All letters are subject to editing and may be shortened. Letters should be sent to the BJGP office by e-mail in the first instance, addressed to

journal@rcgp.org.uk (please include your postal address). Alternatively, they may be sent by post as an MS Word or plain text version on CD or DVD. We regret that we cannot notify authors regarding publication. Letters not published in the Journal may be posted online on our Discussion Forum. For instructions please visit: http://www.rcgp.org.uk/bjgp-discuss

\section{Reflections on a 60-year journey}

Professor John Howie's essay captures many of the problems that were faced by the emerging academic departments of general practice in UK medical schools. As one of those involved over 30 years ago I was only too aware of the 'real doctors' or 'real academics' tension he refers to. After 20 years as a full-time 'real GP' I found myself facing the challenge of setting up academic general practice on a shoestring in a university reluctant to do it. Critically important was the support of local GP colleagues who were willing and enthusiastic to teach, initially for peanuts. This and the appreciation by students of the relevance and value of this teaching ensured survival in hard times. As Howie says, it is encouraging and satisfying to see how far academic general practice has progressed.

\section{Godfrey Fowler,}

Emeritus Professor of General Practice, University of Oxford, 13 Squitchey Lane, Oxford, OX2 7LD.

E-mail: godfrey.fowler@balliol.ox.ac.uk

\section{REFERENCE}

1. Howie J. Academic general practice: reflections on a 60-year journey. Br J Gen Pract 2010; 60(577): $620-622$.

DOI: 10.3399/bjgp10X515421

\section{Two-week cancer referrals: what do you tell the patient?}

All GPs are familiar with the 2-week cancer referral system that has been in place for more than 10 years, and the
NHS Constitution (2010), ${ }^{1}$ but the ways in which individual GPs explain the process to patients differs widely.

Some GPs will explain the potential, albeit sometimes small, risk of cancer to their patients before making the referral, whereas others make no mention of the possible diagnosis at all so as not to 'worry' the patient in advance.

There are a number of potential problems associated with the latter approach. A significant number of patients arrange a consultation with their GP because they are concerned that their symptoms may have a sinister underlying cause and, even if this is not mentioned during the consultation, will remain fearful of a potential cancer diagnosis.

A number of patients appear to be unaware of their GPs' concerns, and in a recent survey of over 160 adult patients, this equated to $49 \% .^{2}$ Anecdotal evidence suggests that some of these patients then did not attend, or in some cases try to delay, their 2-week appointment for up to 3 months, 'choosing' to prioritise work or holiday commitments.

Of more significance are those patients who are shocked or upset when they are contacted by the hospital, prior to their initial appointment, and cancer is mentioned, verbally or in writing.

There is no 'one size fits all' approach to this delicate issue, but it is possible to find a form of words that acknowledges the reason the patient consulted their GP in the first place, recognises the potential for cancer to be among the differential diagnoses, and also highlights, in a positive way, the fact that there is now a system in place that will allow rapid assessment and diagnosis that in $90 \%$ of cases will result in a non-cancer diagnosis. ${ }^{3-5}$ Using this approach, irrespective of the differential diagnosis, the patient will, in most instances, appreciate that serious consideration has been given to their concerns and they have had an opportunity to discuss this openly.

Although the GP may be reassuring during a consultation, it is often worthwhile suggesting that the patient take a friend or family member with them to their hospital appointment. In this way, cancer patients will be more likely to have someone with them if they are given bad news ( $74 \%$ versus $45 \%)$.

During the consultation it is also vital that the GP checks the demographics with the patient, particularly with regard to telephone and mobile phone numbers, as the hospital will more often than not telephone the patient to arrange their appointment.

By adopting this suggested approach, it is possible to improve the experience of people being referred under the 2week rule.

Relton Cummings,

GP Cancer Lead, Newcastle Primary Care Trust.

\section{Maria Vincent,}

Nurse Consultant, Cancer Services/Trust Macmillan Lead Cancer Nurse, Newcastle upon Tyne Hospitals NHS FT.

E-mail: Maria.Vincent@nuth.nhs.uk

\section{REFERENCES}

1. Department of Health. The NHS Constitution: securing the NHS today for generations to come (Cancer). London: Department of Health, 2010.

http://webarchive.nationalarchives.gov.uk/+/www.dh.g ov.uk/en/Healthcare/Cancer/index.htm (accessed 6 Aug 2010)

2. The Newcastle Upon Tyne Hospitals NHS Foundation Trust. Patient experience survey. Cancer services. Newcastle upon Tyne: The Newcastle upon Tyne Hospitals NHS Foundation Trust, 2009.

3. Potter S, Govindarajulu S, Shere M, et al. Referra patterns, cancer diagnoses, and waiting times after introduction of two week wait rule for breast cancer: prospective cohort study. BMJ 2007; 335(7614): 288 .

4. Thorne K, Hutchings H, Elwyn G. The effects of the Two-Week Rule on NHS colorectal cancer diagnostic 
services: a systematic literature review. BMC Health Serv Res 2006; 6: 43.

5. McKie C, Ahmad UA, Fellows S, et al. The 2-week rule for suspected head and neck cancer in the United Kingdom: referral patterns, diagnostic efficacy of the guidelines and compliance. Oral Oncol 2008; 44(9): 851-856.

DOI: 10.3399/bjgp10X515430

\section{Asthma inhalers and colour coding: universal dots}

The importance of colour-coded asthma treatment in patient education is well accepted. Traditionally, reliever medication inhalers are blue in colour and preventer inhalers brown. ${ }^{1}$ This custom is not always followed and the inconsistencies in the colour of inhalers create a lot of confusion.

Marketing of the same medication in different colour inhalers and introduction of new drugs and combinations contributed to this predicament. The confusion created by the introduction of generic salbutamol in UK in orangecoloured inhalers has been previously discussed. ${ }^{2}$ Salbutamol in some countries comes in white inhalers with a blue cap.

The colour coding of caps is never reliable as patients can easily change these stoppers.

Across the world, inhaled corticosteroids (ICS) are available as brown, white, magenta, blue, orange brown, and red inhalers. Long-acting betaagonists (LABA) are now marketed in green, blue, white, and greenish blue containers. The new hydrofluoroalkane propellant-based salbutamol is marketed in red or yellow jackets. Interestingly, their advertisement in a journal asks patients to reach for the 'red' in case of need. Inhaled steroids and LABA combinations were introduced in violet, red, and brown inhalers. So at any point of time a patient can be exposed to a collection of coloured inhalers with total uncertainty of its contents.

Ideally, to have certain colours universally accepted would be most

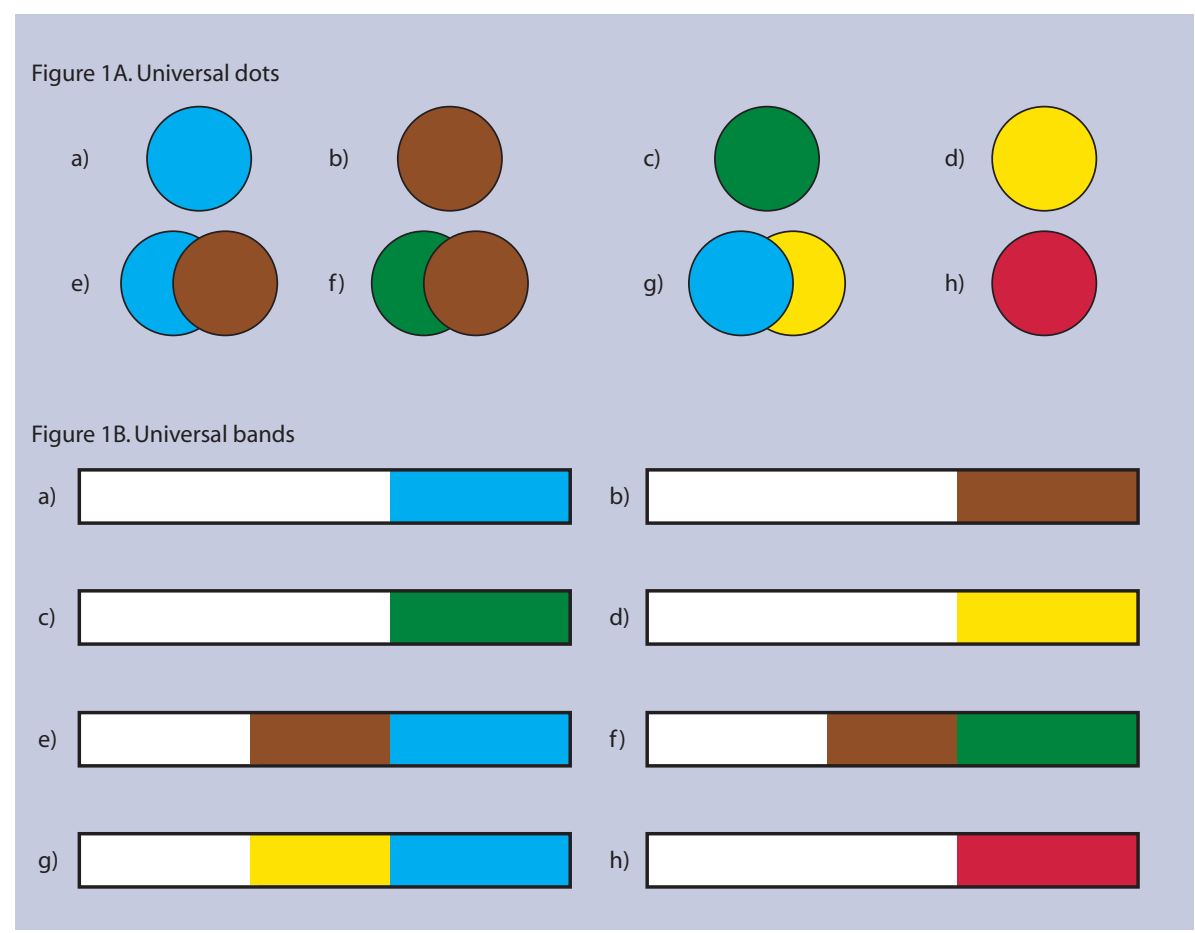

Figure 1A and 1B. a) Blue for beta-agonists. b) Brown for inhaled corticosteroids (ICS). c) Green for long-acting beta-agonists. d) Yellow for anticholinergics. e) Blue and Brown for beta-agonists and ICS combination. f) Green and Brown for long-acting beta-agonists and ICS combination. g) Blue and Yellow for beta-agonists and anticholinergic combination. h) Red to keep in reserve.

effective. But which colour can be used when drugs are marketed in combination? We recommend embedding on the inhalers, small coloured circles or dots. Colours can be assigned to different types of medications. Depending on the contents, the corresponding coloured dot should be placed on the inhaler. When it contains two medications the matching colour dots should partly overlap (Figure 1A). Following the tradition, acceptance will be facilitated by using blue for relievers and brown for inhaled steroids. We suggest green for LABA, yellow for anticholinergics, and black for long-acting anticholinergics. Consequently, one can easily make out that an inhaler with a blue dot contains a bronchodilator irrespective of the fact of where it was purchased, be it in London or in Muscat.

Two overlapping coloured circles, one brown and the other green, convey that the inhaler contains ICS and LABA. Red can be kept aside for future use. We suggest that these identifiers be called ' $U$ dots' (universal inhaler dots). To help patients with visual impairment, ${ }^{3}$ dots should have a slightly raised level with the order of colours reserved universally. Following the same principle, an alternative identification, colour bands, can also be considered; these could be called 'universal bands' or 'U bands' (Figure 1B)

Partridge ${ }^{4}$ narrates his unsuccessful efforts to get responsible bodies interested in the problems created by the introduction of a generic salbutamol inhaler. He is not alone in raising the same concerns. ${ }^{1,5}$ Nevertheless, we hope to revive discussion around the convenience of having uniform colours for inhalers. We call upon all manufacturers to consider this concept and adopt the suggested colours for each class of medications.

\section{B Jayakrishnan,}

Consultant, Department of Medicine, Sultan Qaboos University, PO Box 35, Al Khod, Muscat, Oman.

E-mail: drjayakrish@hotmail.com

Omar A Al-Rawas,

Department of Medicine, Sultan Qaboos University Hospital and College of Medicine and Health Sciences, Oman. 